\title{
ARID1A loss is prevalent in endometrial hyperplasia with atypia and low-grade endometrioid carcinomas
}

\author{
Henrica MJ Werner ${ }^{1,2}$, Anna Berg ${ }^{1,2}$, Elisabeth Wik ${ }^{1,2}$, Even Birkeland ${ }^{2}$, Camilla Krakstad ${ }^{2}$, \\ Kanthida Kusonmano ${ }^{2,3}$, Kjell Petersen ${ }^{3}$, Karl H Kalland ${ }^{4,5}$, Anne M Oyan ${ }^{4,5}$, \\ Lars A Akslen ${ }^{4}$, Jone Trovik ${ }^{1,2}$ and Helga B Salvesen ${ }^{1,2}$ \\ ${ }^{1}$ Department of Obstetrics and Gynecology, Haukeland University Hospital, Bergen, Norway; ${ }^{2}$ Department \\ of Clinical Medicine, University of Bergen, Bergen, Norway; ${ }^{3}$ Computational Biology Unit, Uni Computing, \\ Uni Research AS, Bergen, Norway; ${ }^{4}$ Section For Pathology, The Gade Institute, University of Bergen, Bergen, \\ Norway and ${ }^{5}$ Department of Microbiology, Haukeland University HospitalBergen, Norway
}

\begin{abstract}
ARID1A (AT-rich interactive domain 1A) has recently been identified as a tumor suppressor gene in various, predominantly gynecological cancers. We wanted to investigate the distribution of ARID1A in endometrial hyperplasia, carcinomas and metastatic lesions to elucidate the timing of expression loss of its protein ARID1A in the course of endometrial cancer carcinogenesis. In addition, we wanted to assess the relationship between the loss of ARID1A and clinicopathological variables in endometrial cancer in general and the endometrioid subtype in particular. We analyzed a prospectively collected series of 535 primary endometrial cancers, 77 metastatic lesions, as well as 38 retrospectively collected endometrial hyperplasias with evaluable immunohistochemical staining for ARID1A. Fresh frozen tissue was available for mRNA microarray analysis in 122 primary tumors in parallel. Loss of ARID1A protein expression was noted in none of the hyperplasias without atypia, $16 \%$ of hyperplasias with atypia, $19 \%$ of primary endometrioid tumors and $28 \%$ of metastatic lesions. Loss of ARID1A in primary tumor was significantly associated with endometrioid grade 1 or 2 and clearcell histology, diploid tumor cells, younger patient age and deeper myometrial infiltration, but not survival. ARID1A RNA expression was significantly correlated with ARID1A protein loss. Thus, loss of ARID1A appears to be an early event in the carcinogenesis of endometrioid uterine carcinomas and the association with deep myometrial infiltration may suggest an importance for invasiveness.
\end{abstract}

Modern Pathology (2013) 26, 428-434; doi:10.1038/modpathol.2012.174; published online 19 October 2012

Keywords: ARID1A; carcinoma; endometrial

Endometrial cancer is the pelvic gynecological cancer with the highest incidence in the western world, affecting 24.7/100000 women annually., ${ }^{1,2}$ There are currently few reliable markers to support clinicians in their treatment choice or to help unravel the carcinogenic process in this cancer.

Two independent studies, published simultaneously in 2010, identified ARID1A (AT-rich interactive domain $1 A$ ) as a possible tumor suppressor gene with frequent mutations, leading to loss of

Correspondence: HMJ Werner, MD, Department of Obstetrics and Gynecology, Haukeland University Hospital, Jonas Liesvei 72, Bergen 5058, Norway.

E-mail: heaw@helse-bergen.no

Received 2 July 2012; revised 8 August 2012; accepted 8 August 2012; published online 19 October 2012
ARID1A protein expression in ovarian clear-cell and endometrioid cancers (30-57\%). . $^{3,4}$

ARID1A is located on chromosome 1p36.11 and encodes a protein that is a key component of the SWI/SNF (switch/sucrose non-fermenting) chromatin remodeling complex in eukaryotes. The SWI/ SNF complex is involved in the regulation of essential processes, such as differentiation, proliferation, DNA repair and tumor suppression. The complex mobilizes nucleosomes in an ATPdependent manner to alter the accessibility of the promoters, enhancing or repressing transcription. ARID1A is thought to confer specificity to the regulation of gene expression in this process. ${ }^{5-7}$ Mutations in various subunits of the SWI/SNF can influence the epigenetic regulation of genes and have been identified in different cancers in recent years. ${ }^{5,6}$ 
Since the two reports on ARID1A in 2010, high frequencies of mutations in the ARID1A gene and loss of ARID1A protein have been reported in endometrial cancer $(26-37 \%), 8,9$ adenocarcinoma of the cervix $(32 \%),{ }^{10}$ gastric cancer $(10-14 \%),{ }^{9,11}$ anaplastic thyroid cancer $(14 \%),{ }^{9}$ colorectal cancer $(10 \%),{ }^{11}$ breast cancer $(64 \%)^{12}$ and several cancer cell lines. ARID1A mutations seem, although also present in other tumor types, predominantly linked to gynecological and in particular endometriumrelated tumors.

It has now been shown that $A R I D 1 A$ is a bona fide tumor suppressor gene in gynecological cancers, among others in an interesting study by Guan et $a l^{13,14}$ as was already suggested in many reports. ${ }^{3,4,6}$

We have investigated the expression of ARID1A in endometrial cancer, in the complete spectrum from precursor lesions (hyperplasias), via primary tumors to metastases, to gain a better understanding in the timing of the loss of ARID1A in endometrial cancer carcinogenesis and progression. In addition, we wanted to assess the relationship between loss of ARID1A and clinicopathological variables in endometrial cancer in general and in the endometrioid subtype in particular.

\section{Materials and methods}

\section{Patient Series}

Endometrial Cancer Patients. In total, 641 patients with endometrial cancer were consecutively and prospectively included in our database from May 2001 to November 2011 and treated at the department of gynecology and obstetrics, Haukeland University Hospital, Bergen, Norway. Clinicopathological data including age at diagnosis, FIGO 2009 stage ${ }^{15}$ histological subtype, grade and treatment were recorded as previously reported $^{16}$ and formalin-fixed paraffin-embedded tumor tissue was collected from all patients. Patients were routinely subjected to hysterectomy and bilateral salpingooophorectomy after obtaining a histological diagnosis of endometrial cancer, unless surgery was contra-indicated due to severe co-morbidity. Follow-up data regarding recurrence and survival were provided by primary physicians and private gynecologists responsible for outpatient follow-up, as well as the hospital patient records. Date of last follow-up entry was January 2012. The mean followup was 30 months (median 24 months, range 0-111).

In addition, we studied 77 metastases (formalinfixed paraffin-embedded tumor tissue) of which 71 were paired with corresponding primary tumor. In six patients we only had tissue from the metastasis available.

\section{Hyperplasia Patients}

The hyperplasia series consists of a retrospectively collected series of patients who were diagnosed with endometrial hyperplasia at Haukeland university hospital and underwent hysterectomy and bilateral salpingooophorectomy for that reason between 2001 and 2009 . Clinical-pathological information was collected as indicated above. Patients with hyperplasia were not subjected to follow-up visits. A total of 38 patients were included in this series, of which 31 had complex hyperplasia with atypia.

\section{Tissue MicroArray}

Tissue microarrays (TMAs) were generated as previously described and validated in several studies. ${ }^{17-19}$ In short, the area of highest tumor grade in primary tumor or hyperplasia was identified on H\&E-stained slides, and three (primary tumor and hyperplasia) or one (metastasis) tissue cylinders were punched out from these areas and mounted into a new, recipient paraffin block using a custom-made precision instrument (Beecher Instruments, Silver Spring, MD, USA).

\section{Immunohistochemistry}

Tissue microarray sections $(5 \mu \mathrm{m})$ were dewaxed with xylene and rehydrated in ethanol before microwave target retrieval (350 Watt; $20 \mathrm{~min}$ ) in TRS buffer $\mathrm{pH}-6$. Slides were subsequently blocked for peroxidase and incubated at room temperature with primary monoclonal antibody (ARID1A, Biosite: AT1188a) 1:100 for $2 \mathrm{~h}$. Envision HRPlabeled anti-mouse (30 min, room temperature) was applied as secondary antibody. Finally, slides were counter-stained with Dako hematoxylin. Evaluable staining for ARID1A was available for 535 primary tumors mounted in TMA.

Blinded for patient characteristics and outcome, slides were scored by two of the authors (HMJW and JT), by standard light microscope, as described previously in more detail. $^{20,21}$ In short, a semi-quantitative scoring system was applied, considering intensity of staining and the proportion of tumor cells with this intensity. The score was the product of intensity, graded from 0 (no staining) to 3 (intense staining) and an area of tumor with this staining intensity, graded from 0 (no positive tumor cells) to 3 ( $>50 \%$ positive tumor cells) with a final score $0-9$. In accordance with the article published in New England Journal of Medicine, ${ }^{4}$ tumors were scored positive if tumor cells showed any nuclear staining (score 1-9) and negative if tumor cells stained completely negative (score 0 in our scoring system), but showed positive stromal/non-tumor cells in the same patient (internal control). As all normal tissue showed immunoreactivity for ARID1A; cases in which both normal and tumor tissue were negative were considered technical failure and, therefore, excluded from analysis. A subset of the cases $(n=63)$ was scored independently by the above two authors (JT and HMJW) to calculate the inter-observer variability.

\section{RNA Microarray Analysis}

RNA was extracted from 122 fresh frozen primary tumors (RNeasy Mini Kit, Qiagen, Hilden, Germany) and 
hybridized to Agilent Whole Human Genome Microarrays $44 \mathrm{k}$ (Cat. no. G4112F), according to the manufacturer's instructions. Three different probes were available, known to attach to different areas of the ARID1A gene (Agilent: A_24_P92952, A_23_P217872, A_24_P77219), of which expression levels for the first two were highly significantly correlated, thus averaged and applied for further analyses. Arrays were scanned and features extracted using the Agilent Microarray Scanner Bundle. Mean spot signals were used as intensity measure and expression data were normalized using median over entire array. Raw data were imported and analyzed using the J-Express software (Molmine, Norway). Gene expression data were uploaded to the Array Express database according to MIAME guidelines (Accession number pending).

\section{Statistical Analysis}

Statistical analysis was performed with PASW 18 Statistics (Predictive Analysis SoftWare, SPSS inc. Chicago, USA). Kaplan-Meier curves were generated to evaluate disease-specific survival. Statistical significance of the association between ARID1A and clinical-pathological variables was assessed by the $\chi^{2}$-test. The non-parametric Mann-Whitney U-test was used to correlate protein and RNA expression data. Two-sided $P$-values of $\leq 0.05$ were considered significant.

\section{Approvals and Grant Support}

The study has been approved by the Norwegian Data Inspectorate (961478-2), the Norwegian Social Science Data Services (15501) and the local Institutional Review Board (REKIII nr. 052.01). The study has been supported by The Norwegian Cancer Society (The Harald Andersen's legacy), Helse Vest Research Fund, Norwegian Research Council and The University of Bergen Meltzer Foundation.

\section{Results}

From the 641 patients included, evaluable immunohistochemistry staining results for ARID1A were available in 535 patients. The patients with and without staining results available were similar in their distribution of demographical variables, supporting no systematic bias (data not shown). Table 1 shows the demographics of the 535 patients with available immunohistochemistry data.

ARID1A staining was nuclear, both in stromal and glandular tissue. Expression in stromal tissue was used as internal positive control as described in the Material and Methods section. All normal tissue showed moderate to intense staining for ARID1A.

Loss of ARID1A, defined by a total loss of expression in tumor cells, was noted in none of the hyperplasias without atypia, $16 \%$ of the complex hyperplasias with atypia, $19 \%$ of primary tumors of endometrioid subtype and $28 \%$ of metastatic lesions (Figure 1). From hyperplasia with
Table 1 Characteristics of the 535 endometrial cancer patients with ARID1A immunohistochemical scoring available

\begin{tabular}{|c|c|}
\hline Variable & Patients with ARID1A scoring n (\%) \\
\hline Patients & 535 \\
\hline \multicolumn{2}{|l|}{ FIGO stage } \\
\hline FIGO stage 1 & $412(77)$ \\
\hline FIGO stage 2 & $36(7)$ \\
\hline FIGO stage 3 & $62(12)$ \\
\hline FIGO stage 4 & $25(5)$ \\
\hline \multicolumn{2}{|l|}{ Histological subtype } \\
\hline Endometrioid & $436(81.5)$ \\
\hline Non-endometrioid & 99 (19) \\
\hline Clear-cell & $19(4)$ \\
\hline Serous & $44(8)$ \\
\hline Carcinosarcoma & $26(5)$ \\
\hline Undifferentiated & $10(2)$ \\
\hline \multicolumn{2}{|l|}{ Differentiation grade ${ }^{\mathrm{a}}$} \\
\hline 1 & $192(36)$ \\
\hline 2 & $166(31)$ \\
\hline 3 & $173(33)$ \\
\hline \multicolumn{2}{|l|}{ Lymphadenectomy } \\
\hline Performed & $439(82)$ \\
\hline Not performed & 96 (18) \\
\hline \multicolumn{2}{|l|}{ Recurrence } \\
\hline Yes & $87(16)$ \\
\hline No & $412(77)$ \\
\hline Not radically operated & $36(7)$ \\
\hline \multicolumn{2}{|l|}{ Menopausal status } \\
\hline Pre-/peri-menopausal & $65(12)$ \\
\hline Post menopausal & $470(88)$ \\
\hline \multicolumn{2}{|l|}{ Primary treatment } \\
\hline Hysterectomy & $533(100)$ \\
\hline Tumor reduction & $1(0)$ \\
\hline Curettage only & $1(0)$ \\
\hline \multicolumn{2}{|l|}{ Myometrial infiltration ${ }^{\mathrm{b}}$} \\
\hline$<50 \%$ & $340(64)$ \\
\hline$>50 \%$ & $193(36)$ \\
\hline \multicolumn{2}{|c|}{ Primary lymph node metastasis } \\
\hline Yes & $51(10)$ \\
\hline No & $387(72)$ \\
\hline Not examined & $97(18)$ \\
\hline \multicolumn{2}{|l|}{ DNA ploidy ${ }^{\mathrm{C}}$} \\
\hline Diploid & $285(79)$ \\
\hline Aneuploid & $76(21)$ \\
\hline Age (median) & $65.4(28-93)$ \\
\hline BMI (median) & $28.2(14.7-73.0)$ \\
\hline
\end{tabular}

Abbreviations: BMI: body mass index; FIGO: International Federation of Gynecology and Obstetrics.

${ }^{\mathrm{a}}$ Four cases missing.

${ }^{\mathrm{b}}$ Two cases missing.

${ }^{\mathrm{C}} 153$ cases missing.

atypia to primary tumor (endometrioid histology) alone, the difference was not statistically significant ( $P$-value 0.616). Paired testing of the 71 cases with primary tumor and metastasis showed borderline statistical significance (0.054). Representative 


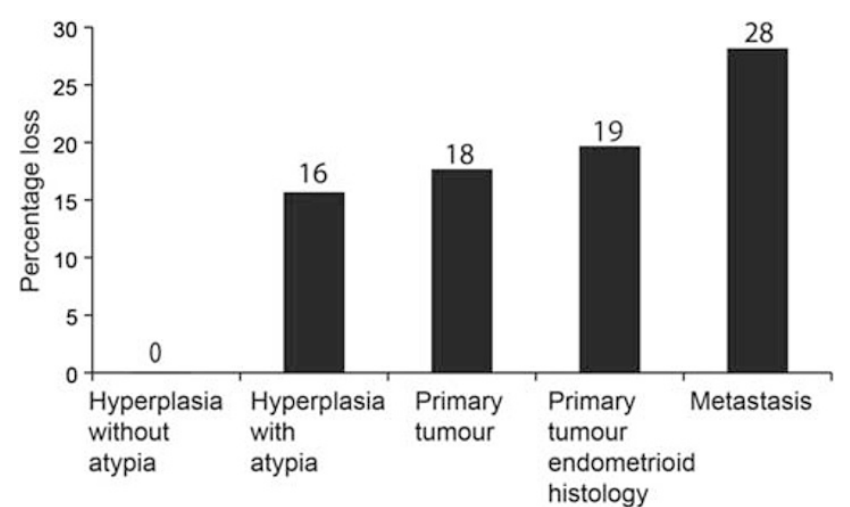

Figure 1 Increase in loss of ARID1A from hyperplasia via primary tumor to metastasis.
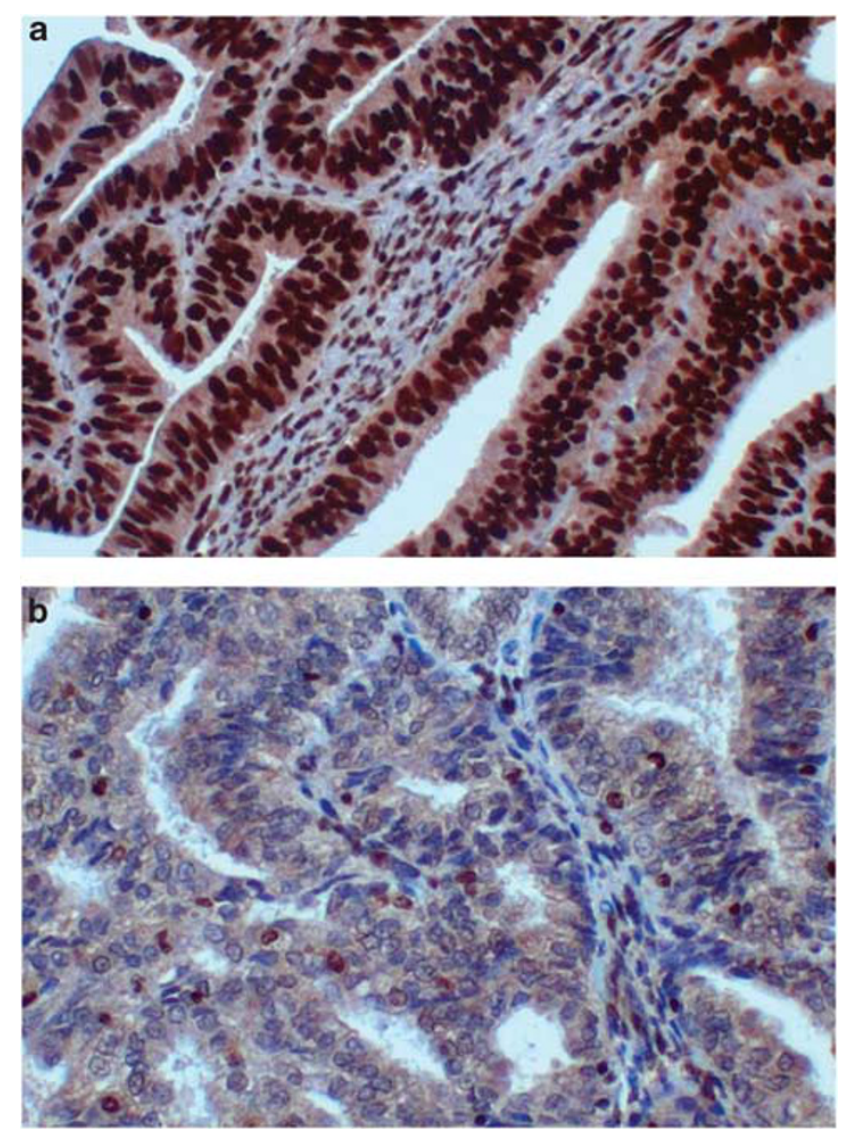

Figure 2 Examples of the immunohistochemical staining showing (a) Positive ARID1A expression. (b) Loss of ARID1A expression.

images of positive and negative staining are shown in Figure 2. The $\kappa$-value for inter-observer reproducibility of ARID1A staining (HMJW and JT) was 0.79 .

Loss of ARID1A was significantly associated with endometrioid histology $(19 \% ; 84 / 436$, Table 2$)$, as well as clear-cell histology $(21 \% ; 4 / 19)$. In contrast, only $3 \%$ of the serous tumors were ARID1A negative (1/44).
Loss of ARID1A was also significantly associated with younger age, grade 1 and 2 in endometrioid tumors, deep myometrial invasion and diploid cancer cells as shown in Table 2.

No relationship was found between ARID1A loss and disease-specific survival (Figure 3) nor progression-free survival (not shown). In the subgroup of endometrioid histology only, a significant relationship exists between ARID1A loss and higher FIGO stages (FIGO $3-4, P=0.04$ ). Considering the entire population (including non-endometrioid cases) there is no significant relationship with FIGO stage. We did not find any significant relationship between ARID1A and pathological p53 expression as reported by others, ${ }^{13}$ loss of estrogen or progesterone receptor ${ }^{12}$ in either the entire population or the endometrioid histology subgroup (data not shown).

Levels of mRNA expression for ARID1A were statistically significantly correlated with ARID1A protein expression $(P=0.01)$. In line with the correlations seen for protein expression, we found a significantly lower mRNA level for ARID1A in tumors with endometrioid histology $(P=0.02)$ and a tendency to association with deep myometrial invasion $(P=0.06)$.

To explore if deep myometrial invasion could be explained by a link between ARID1A and epithelial to mesenchymal transition (EMT), we examined if two gene signatures for $\mathrm{EMT}^{22}$ related to ARID1A mRNA and protein expression levels. No such relation was identified to support EMT activation as a mechanism for the observed deeper myometrial invasion with ARID1A loss.

\section{Discussion}

ARID1A is a recently discovered marker, associated with predominantly gynecological cancers. ${ }^{3,4,8-11,23}$ In the study by Wiegand, ${ }^{9}$ as well as in the study by Guan, ${ }^{8}$ it has been shown that ARID1A mutations are strongly correlated with loss of ARID1A by immunohistochemistry in both the ovarian and endometrial carcinomas. ${ }^{8,9}$ Mamo did also find an association between DNA copy-number changes in ARID1A and protein expression but considered that these changes could not account for all ARID1A loss in breast cancer tumors. ${ }^{12}$

Literature on ARID1A shows considerable variation in antibodies, protocols and scoring methods used. The two articles concerning endometrial cancer $^{8,9}$ used different types of ARID1A antibodies, Abgent monoclonal mouse antibody during $2 \mathrm{~h}$ at room temperature vs Sigma Aldrich polyclonal antibody overnight. They applied the same scoring cutoff; completely negative vs any positive ARID1A staining of epithelial cells.

In studies of cervical-, ovarian- and breast-cancers, two authors ${ }^{10,12,23}$ reported a correlation between ARID1A and more aggressive clinical features both using a Santa Cruz monoclonal 
Table 2 ARID1A protein expression related to clinicalpathological variables

\begin{tabular}{|c|c|c|c|}
\hline \multirow{2}{*}{ Variable } & \multicolumn{2}{|c|}{ ARID1 $A^{\mathrm{a}}$} & \multirow{2}{*}{ P-value } \\
\hline & $\begin{array}{l}\text { Loss, } \\
\mathrm{n}(\%)\end{array}$ & $\begin{array}{c}\text { No loss, } \\
\text { n (\%) }\end{array}$ & \\
\hline Histological subtype & & & 0.031 \\
\hline Endometrioid & $84(19)$ & $352(81)$ & \\
\hline Non-endometrioid & $10(10)$ & $89(90)$ & $0.049^{\mathrm{b}}$ \\
\hline Clear-cell & $4(21)$ & $15(79)$ & \\
\hline Serous & $1(2)$ & $43(97)$ & \\
\hline Carcinosarcoma/undiff & $5(14)$ & $31(86)$ & \\
\hline Differentiation (endometrioid only) ${ }^{\mathrm{C}}$ & & & 0.007 \\
\hline 1 & $26(14)$ & $166(87)$ & \\
\hline 2 & $44(27)$ & $122(74)$ & \\
\hline 3 & $13(18)$ & $61(82)$ & \\
\hline Menopausal status & & & 0.583 \\
\hline Pre-/peri-menopausal & $13(20)$ & $52(80)$ & \\
\hline Post menopausal & $81(17)$ & $389(83)$ & \\
\hline Myometrial infiltration ${ }^{\mathrm{d}}$ & & & $<0.001$ \\
\hline$<50 \%$ & $44(13)$ & $296(87)$ & \\
\hline$>50 \%$ & $48(25)$ & $145(75)$ & \\
\hline DNA ploidy ${ }^{\mathrm{e}}$ & & & 0.002 \\
\hline Diploid & $58(20)$ & $227(80)$ & \\
\hline Aneuploid & $4(5)$ & 72 (95) & \\
\hline Age & & & 0.005 \\
\hline Under median (66 years) & $64(22)$ & $231(78)$ & \\
\hline Over median (66 years) & $30(13)$ & $210(88)$ & \\
\hline FIGO & & & 0.253 \\
\hline Stage I-II & $75(17)$ & $373(83)$ & \\
\hline Stage III-IV & $19(22)$ & $68(78)$ & \\
\hline
\end{tabular}

${ }^{\mathrm{a}}$ ARID1A loss is defined as score $=0$; no loss as score $=1-9$.

bIn the subgroup of the non-endometrioid subtypes.

${ }^{\mathrm{C}}$ Four cases missing.

${ }^{\mathrm{d}}$ Two cases missing.

$\mathrm{e}_{174}$ cases missing.

antibody. Mamo, ${ }^{12}$ however, included slight positive ARID1A stain in the 'negative' group whereas Katagiri ${ }^{10,23}$ scored negative only those cases that were completely negative for ARID1A.

The mutations identified in the ARID1A gene $^{3,4,8,12}$ are supposed to be truncating. The epitopes, recognized by the various antibodies in IHC vary. One can therefore hypothesize that an antibody, recognizing an epitope earlier in the protein shows more immunoreactivity in mutated cases than another antibody, recognizing an epitope later in the protein as the protein gets truncated. This, as well as other differences in staining protocol and scoring cutoff applied can possibly account for some variation between studies on ARID1A. For this reason we chose to use the same antibody and scoring cutoff as Wiegand et al did in their article, ${ }^{4}$ considered to be the most comprehensive study available.

Consistent with the literature, we noted that loss of ARID1A is most frequent in the endometrioid and
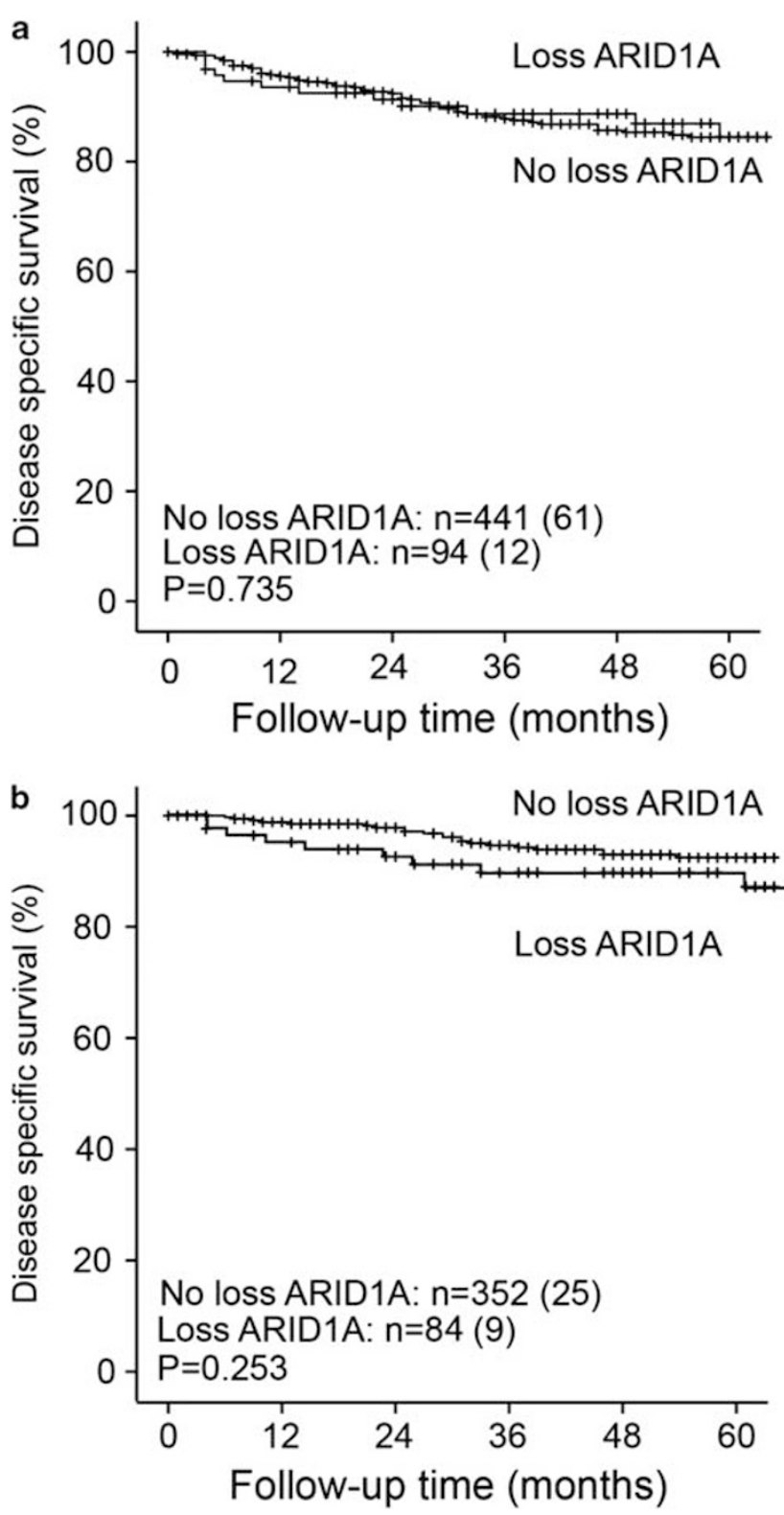

Figure 3 Disease-specific survival for patients with endometrial carcinoma (Kaplan-Meier) related to expression of ARID1A protein. (a) All tumors (b) endometrioid subtype only.

clear-cell histological subtypes, but a much less frequent event in serous tumors. ${ }^{4,8,9}$ We noted that ARID1A loss is also associated with younger age and diploid tumor cells, therefore related to less aggressive endometrial tumors. Our finding of ARID1A loss correlating with grade 1 and 2 differentiations also appears to be in line with this. Apparently contrasting it, both in the entire population and the endometrioid subgroup, loss was significantly associated with deeper myometrial infiltration, although this was not translated in a reduced disease-specific survival. This suggests that ARID1A loss, observed also in hyperplasia with atypia, may be related to the development of 
invasiveness. Further studies are needed to explore this observation more in detail.

The lack of correlation between ARID1A and disease-specific survival, also reported by some other authors, ${ }^{4,10}$ may also be explained if ARID1A mutation is important in early stages of the carcinogenesis rather than tumor progression. Another explanation may be that mutations in other chromatin remodeling genes have a role in those tumors that are positive for ARID1A, obscuring any effects in the ARID1A negative group. In endometrial carcinomas this was previously unexplored, as neither Wiegand nor Guan did look into clinical-pathological variables in their endometrial cancer series. ${ }^{9,13}$ In ovarian clear-cell and breast cancer, a relation between clinical-pathological variables and loss of ARID1A expression was noted by Katagiri and Mamo, respectively, ${ }^{12,23}$ where in both ovarian clear-cell carcinoma and breast cancer loss of ARID1A was related to some aggressive features including higher FIGO stage in the ovarian clear-cell carcinoma. ${ }^{23}$

In our patient series we are able to show a highly significant correlation between loss of protein and mRNA expression data, further supporting that immunohistochemistry can be used as a proxy for ARID1A status in endometrial carcinomas.

It is known that endometrioid endometrial tumors arise from hyperplastic endometrium whereas serous tumors originate from atrophic endometrium. ${ }^{2,24}$ We found loss of ARID1A in none of the hyperplasias without atypia and $16 \%$ of the complex hyperplasias with atypia, the latter considered to be the precursor of endometrioid endometrium carcinoma. Wiegand noted loss of ARID1A in atypical endometriosis adjacent to tumor, considered the precursor for ovarian endometrioid and clear-cell tumors, but not in a small set (9 cases) of complex atypical hyperplasia. ${ }^{9}$ Mamo $^{12}$ noted ARID1A loss in precancerous lesions in the breast in $37-52 \%$ (normal epithelial cells and carcinoma in situ) and a higher percentage in invasive carcinoma (64\%) and metastasis (80\%), underlining that loss of ARID1A may be an early event in the breast cancer carcinogenesis. Our findings and the contrast with only $1 \%$ loss of ARID1A in tumors with serous histology, support that this is an (early) event in endometrioid, but not serous endometrial carcinoma carcinogenesis; the latter most likely defined by other genetic abnormalities and precursor lesions.

\section{Conclusions}

Loss of ARID1A may be an early event in the carcinogenesis of endometrioid uterine carcinomas, supported by the similar proportion of protein loss in the precursor lesion hyperplasia with atypia as in endometrioid primary tumors. We confirm that loss of ARID1A expression is related to endometrioid and clear-cell histology but infrequent in the serous subtype. Loss of ARID1A is associated with differentiation grade 1 and 2 but not with survival, although a significant correlation with deep myometrial infiltration may support an importance for development of early invasiveness.

\section{Acknowledgements}

We thank Gerd Lillian Hallseth for her help with the immunohistochemistry. Britt Edvardsen receives thanks for all her support in the laboratory. Tormund Njølstads help is much appreciated in making the TMA collection.

\section{Disclosure/conflict of interest}

The authors declare no conflicts of interest.

\section{References}

1 Abeler VM, Kjorstad KE. Endometrial adenocarcinoma in Norway. A study of a total population. Cancer 1991;67:3093-3103.

2 Amant F, Moerman P, Neven P, et al. Endometrial cancer. Lancet 2005;366:491-505.

3 Jones S, Wang TL, Shih Ie M, et al. Frequent mutations of chromatin remodeling gene ARID1A in ovarian clear cell carcinoma. Science 2010;330:228-231.

4 Wiegand KC. ARID1A mutations in endometriosisassociated ovarian carcinomas. N Engl J Med 2010;363:1532-1543.

5 Reisman D, Glaros S, Thompson EA. The SWI/SNF complex and cancer. Oncogene 2009;28:1653-1668.

6 Wilson BG, Roberts CWM. SWI/SNF nucleosome remodellers and cancer. Nat Rev Cancer 2011;11: 481-492.

$7 \mathrm{Wu}$ JI, Lessard J, Crabtree GR. Understanding the words of chromatin regulation. Cell 2009;136:200-206.

8 Guan B, Mao TL, Panuganti PK, et al. Mutation and loss of expression of ARID1A in uterine low-grade endometrioid carcinoma. Am J Surg Pathol 2011; 35:625-632.

9 Wiegand KC, Lee AF, Al-Agha OM, et al. Loss of BAF250a (ARID1A) is frequent in high-grade endometrial carcinomas. J Pathol 2011;224:328-333.

10 Katagiri A, Nakayama K, Rahman MT, et al. Frequent loss of tumor suppressor ARID1A protein expression in adenocarcinomas/adenosquamous carcinomas of the uterine cervix. Int J Gynecol Cancer 2012;22: 208-212.

11 Jones S, Li M, Parsons DW, et al. Somatic mutations in the chromatin remodeling gene ARID1A occur in several tumor types. Hum Mutat 2012;33:100-103.

12 Mamo A, Cavallone L, Tuzmen S, et al. An integrated genomic approach identifies ARID1A as a candidate tumor-suppressor gene in breast cancer. Oncogene 2012;31:2090-2100.

13 Guan B, Wang TL, Shih Ie M. ARID1A, a factor that promotes formation of SWI/SNF-mediated chromatin remodeling, is a tumor suppressor in gynecologic cancers. Cancer Res 2011;71:6718-6727. 
14 Huang J, Zhao YL, Li Y, et al. Genomic and functional evidence for an ARID1A tumor suppressor role. Genes Chromosomes Cancer 2007;46:745-750.

15 Creasman W. Revised FIGO staging for carcinoma of the endometrium. Int J Gynaecol Obstet 2009;105:109.

16 Trovik J, Wik E, Stefansson IM, et al. Stathmin overexpression identifies high-risk patients and lymph node metastasis in endometrial cancer. Clin Cancer Res 2011;17:3368-3377.

17 Kononen J, Bubendorf L, Kallioniemi A, et al. Tissue microarrays for high-throughput molecular profiling of tumor specimens. Nat Med 1998;4:844-847.

18 Stefansson IM, Salvesen HB, Akslen LA. Prognostic impact of alterations in P-cadherin expression and related cell adhesion markers in endometrial cancer. J Clin Oncol 2004;22:1242-1252.

19 Voduc D, Kenney C, Nielsen TO. Tissue microarrays in clinical oncology. Semin Radiat Oncol 2008;18:89-97.

20 Engelsen IB, Stefansson IM, Akslen LA, et al. GATA3 expression in estrogen receptor alpha-negative endo- metrial carcinomas identifies aggressive tumors with high proliferation and poor patient survival. Am J Obstet Gynecol 2008;199:e1-e7.

21 Salvesen HB, Das S, Akslen LA. Loss of nuclear p16 protein expression is not associated with promoter methylation but defines a subgroup of aggressive endometrial carcinomas with poor prognosis. Clin Cancer Res 2000;6:153-159.

22 Jechlinger M, Grunert S, Tamir IH, et al. Expression profiling of epithelial plasticity in tumor progression. Oncogene 2003;22:7155-7169.

23 Katagiri A, Nakayama K, Rahman MT, et al. Loss of ARID1A expression is related to shorter progression-free survival and chemoresistance in ovarian clear cell carcinoma. Mod Pathol 2012;25: 282-288.

24 Llaurado M, Ruiz A, Majem B, et al. Molecular bases of endometrial cancer: New roles for new actors in the diagnosis and the therapy of the disease. Mol Cell Endocrinology 2012;25:244-255. 